\title{
Getting Back To Exercise Without Pain: The Ankle part I.
}

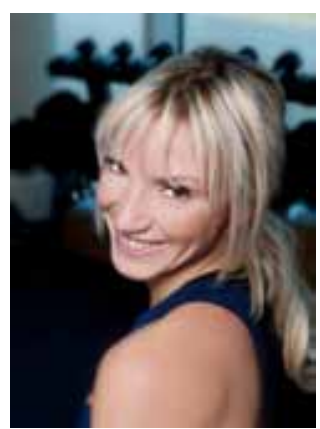

Authors: JUDIT RESSINKA

Title: Personal Trainer, Strength \& Conditioning Coach

Workplace: Crowne Plaza Battersea e-mail: juditressinka@yahoo.co.uk Webpage: www.movepainfree.co.uk Picture, figure: JUDIT RESINKA Other-outhors: PÉTER FRITZ, ZOLTÁN SZATMÁRI

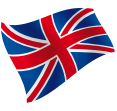

Abstract: Physical activity is becoming an increasingly popular lifestyle choice as people are encouraged to make healthier choices. For most people, this means balancing exercise with a working day that typically requires them to be sitting for 10 to 12 hours which lessens the ability of the body to effectively take part in recreational and sporting activities, and leads to repetitive strain injuries, postural deviations and musculoskeletal problems $(8,16,27)$. Therefore, the desire of the modern client to combine extended periods of inactivity with increasingly demanding exercise routines, such as marathon running, are becoming a growing challenge for personal trainers and sports coaches.

Such issues occur because muscles have the tendency to become underactive or overactive when engaged by certain repetitive postures and activities $(20,27)$. For example, sitting, driving or wearing high heels can encourage the ankle dorsiflexors to weaken, the plantarflexors to become overactive, while it is also suggested that there will be interrelated, global impairments and consequences of this at the more proximal joints such as the knees and hips $(11,12,14,19,23,24,30)$. Achilles tendinopathy $(1,31)$, plantar fasciitis $(18)$ and shin splints $(30,33)$ are some of the most common sports injuries of the ankle among runners and those participating in sports that involve jumping.

As coaches, we shouldn't be diagnosing injuries, but helping to prevent the occurrence of them, by looking for common lower extremity alignments, muscle weaknesses and movement deviations. This article focuses on the evaluation of movement, the scanning of misalignments and the introduction of corrective exercises using the NASM Corrective Exercise Continuum (Cex) (7), which is based on deactivation and activation techniques of the neuromyofactial tissue (7). Using CEx with my clients - in conjunction with body and postural awareness techniques - has enabled them to eliminate some musculoskeletal problems.

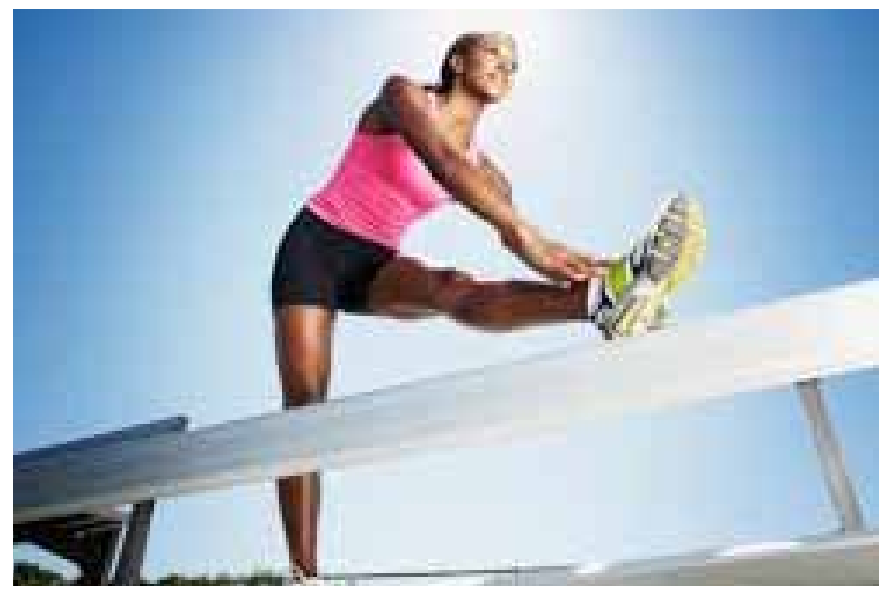

[Figure1]
Keywords: ankle dorsiflexion, medial knee displacement, over-pronation, hip adductor, the NASM Corrective Exercise Continuum,

\section{Questions}

Is it enough to simply look at the ankle's limitations when an actual injury occurs or as with the knee, do we need to apply a global approach and look elsewhere?

What are the signs of ankle limitations - and what is considered to be normal alignment in a preventative point of view? Is there a universal solution for improving limited dorsiflexion and ankle ROM?

\section{Introduction}

Ankle joint stiffness is one of the main causes of lower extremity injuries, degenerative diseases and inflammatory conditions that cause pain and impair normal activities. It usually takes years to develop and often only becomes evident if the client suddenly increases intensity, mileage or increases weight load too quickly.

It comprises both inflexibility of the calf muscles, the gastrocnemius and soleus, and the Achilles tendon, as well as limited movement between the bones of the ankle. It results in decreased ankle dorsiflexion $(1,18,31,33)$, [foot movement towards the shin] (Figure 2) - a risk factor for several overuse-type injuries of the leg $(11,14,21,26,29)$. Resulting ankle and foot pain have a devastating effect on an individual's life as physical activity, balance, walking, running gait and gait speeds are all affected $(13,19)$.

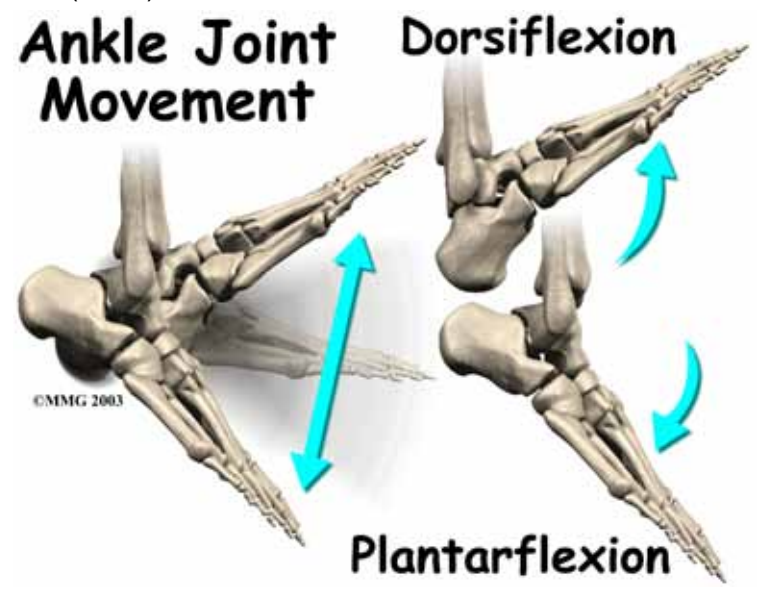

Figure 2. Ankle Joint Movement: plantarflexion and dorsiflexion

There are three factors most likely to contribute to limited ankle mobility; these are: modern footwear, poor posture and limited activity (requiring dorsiflexion).

Dress shoes, boots, heels and even running shoes can elevate the heel and hold the ankle in a plantar flexed position. Over time this causes decrease in dorsiflexion, where the individual loses motionas the weak dorsiflexors are no longer able to pull the foot up towards the shin.

When the spine is in good alignment, the head, shoulders, hips and ankles will form a straight line travelling down along the so-called ,plum line'. Slight variance from this alignment is not likely to be a problem. However, certain postures, such as a forward head posture or an anterior pelvic tilt, significantly alters alignment and causes the body's center of gravity to fall in front of the line (Figure 3). Subsequently, in order to stay 
balanced, the body readjusts and forces the foot in plantar flexion at the ankles. People have a tendency to avoid activities that involve deep squatting and jumping as they age. This prevents the body from using the full range of motion of the ankle. If you don't use and practice full movement of your joints, you will lose the ability to perform them. Simply put, if you don't use it, you lose it.

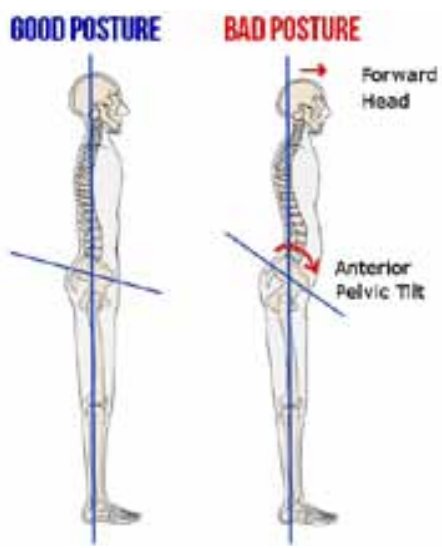

Figure 3: A typical forward head and excessive anterior pelvic tilt posture

\section{The ankle joint}

The unique design of the ankle makes this important hinge joint very stable. With stability, it is able to withstand 1.5 times one's bodyweight when walking and up to eight times one's body weight on running. The joint is formed of three bones: the ankle bone called the talus - which fits into a socket formed by the lower end of the tibia (shinbone) - and the fibula. The bottom of the talus sits on top of the heel bone - the calcaneus. It is the center of the talus, which allows the foot to move up (dorsiflexion) and down (plantarflexion).

Normal ankle function is required to walk smoothly and with an almost effortless gait. A large array of tendons and ligaments make this action possible many thousands of times a day.

The ankle joint's most important tendon in walking, running and jumping is the large Achilles tendon. It attaches the calf muscles (soleus and gastrocnemius) to the heelbone (Figure 4). Therefore, when the calf muscles are contracted, the foot is able to point down and allows us to raise up on to our toes. The posterialtibialis muscle and its tendon attaches to the underside of the foot, allowing the arch to rise and the foot to turn in (inversion) with the contraction. The anterior tibialis at the front of the leg contributes to the dorsiflexion action of the foot as it pulls the foot upward and inverts it (lateral foot edge lowers while medial raises). The peroneals on the outside edge of the ankle do the opposite, so the foot can bend down and turn out in pronation (medial foot edge lowered while the lateral raises).

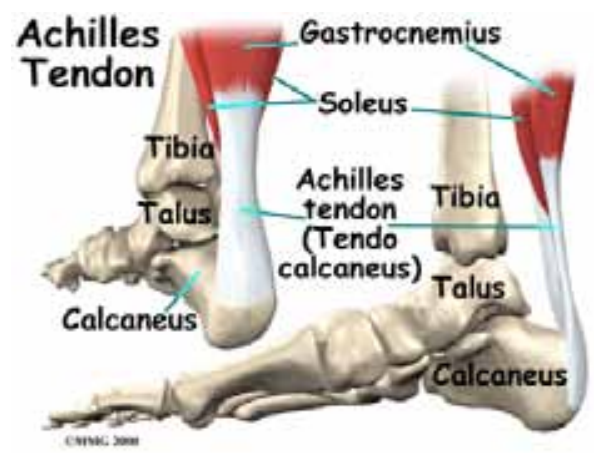

Figure 4. Bones of the ankle joint
The foot is not the only place that the effects of ankle stiffness can be seen. Depending on the level of inflexibility in the ankle, it may cause a complete inability to perform a movement, or create a negative knock-on effect all the way up the posterior chain, with serious potential to cause an injury.

A very likely consequence to this is where other joints try to make up for limited ankle motion and joint alignment is altered e.g. knock knees (excessive knee valgus) toe out walking from ankle stiffness $(3,4,23,24,26)$.

In an athletic context, such ankle limitations severely decrease the athlete's ability to generate maximum force. The loss of neutral positions of joints and the spine impairs the ability to transfer force efficiently from the floor to the hips and to the load.

\section{What are the global factors of excessive knee valgus or medial knee displacement (MKD) and over-pronation?}

The presence of excessive MKD is believed to be influenced by specific strength and flexibility deficits of hip and ankle joint musculature(15). There are possibly dozens of reasons why these ailments occur and cannot be pinpointed to simple one. Let us examine four most-likely causes that influence foot out position, simultaneous over-pronation (flattening of the foot)(Figure 5) and MKD.

Foremost, hip muscle imbalances consisting of hip external rotator and abductor weakness, can enable increased hip internal rotation and adduction motion $(6,7,15,32,34)$, excessive MKD $(3,24,26,33)$, which in turn can cause foot pronation at the bottom of the kinetic chain $(3,11,19,23,24)$.

Similarly, lower-leg muscle imbalances involving tightness of lateral ankle musculature, such as the lateral gastrocnemius and soleus, and peroneals, can contribute to tibial abduction and external rotation, thereby facilitating excessive MKD $(3,4,23,24,26)$.

As the lower leg moves over the foot and if the calf muscles are tight, the ankle reaches its end range of ROM quickly and its only option is to go further forward with the mid-foot arch collapsing. It results in the foot further pronating, now over-pronating, and eventually turning out to gain more range (7)

Furthermore, weakness of the medial gastrocnemius, anterior tibialis, and posterior tibialis may decrease the ability to control knee valgus and foot pronation motions (15).

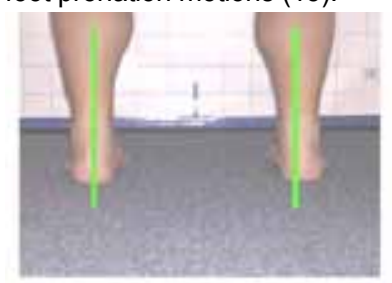

Normal

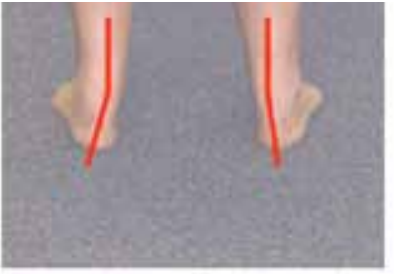

Abnormal
Figure 5. Overpronation of the foot and ankle with the ankle collapsing

\section{Limited ankle dorsiflexion}

To further examine the effect of the ankle on the other joints, several studies have been conducted. On average, a 20 per cent decrease in ankle dorsiflexion ROM has been observed in those who display excessive medial knee displacement (MKD) and dynamic knee valgus collapse, compared with those without displacement during doubleleg squat $(3,4,23,26,34)$, single-leg squat tasks (24) and landing task 
(12) (Figure 6).These collective findings indicate that individuals with restricted passive ankle dorsiflexion ROM, display greater medial knee motion. This additional medial displacement can have devastating effects on the knee joint, contributing to ACLinjuries $(12,14,28)$, patellofemoral knee syndrome $(11,21)$, patellar tendinopathy (2) and several other knee injuries due to the additional compressive forces during movement $(17,25,29)$.

In addition, energy absorption by the knee joint is influenced by ankle dorsiflexion motion (9). For individuals where this is restricted, they may experience a diminished ability to absorb kinetic energy at the ankle (12). This leads to greater weighting on the knee's surrounding soft tissue structures and naturally increases the risk of knee injury.

Therefore, either altered frontal plane knee biomechanics, increased knee joint energy absorption, or both are believed to increase the risk of knee injury (12). As such, ankle dorsiflexion mobility training should be a key component of knee injury prevention programs.
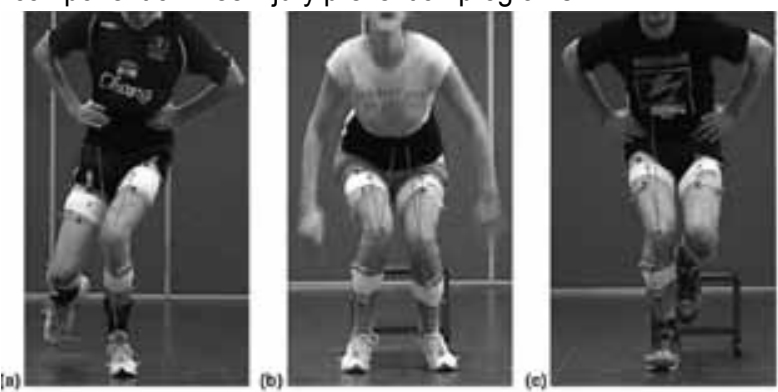

Figure 6. Excessive medial $\mathrm{knee}$ displacement = excessive $\mathrm{knee}$ valgus in single leg squat, (a) and landing (b-c).

\section{Which limitation was first the hips' or the ankle's?}

Heel-lifts using a wedge are used by clinicians to differentiate between lower-leg and hip muscle imbalances (24), which are considered the primary contributing factors to excessive MKD and dynamic knee valgus positioning. If MKD is no longer present after placing a heel-lift under the heel during a double-leg squat, the problem could be found in the calf muscles (Figure 7). Correction of MKD when using a wedge is considered to occur as a result of increasing dorsiflexion range and decreasing tension within the lateral ankle musculature, which restores the normal length-tension relationship of the medial gastrocnemius, anterior tibialis, and posterior tibialis.

If MKD is still present during the double leg squat, even after placement of the heel-lift, then the hip muscle imbalances may be the primary cause. Earl et al, 2011 prescribed a hip-strengthening program to a group of healthy female runners for eight weeks. In addition to their showing a predictable increase in hip strength at the end of the program, the runners also exhibited significantly less pronation (measured by how far the heel collapsed inwards). In all, the participants experienced 57 per cent less pronation at the ankle joint.

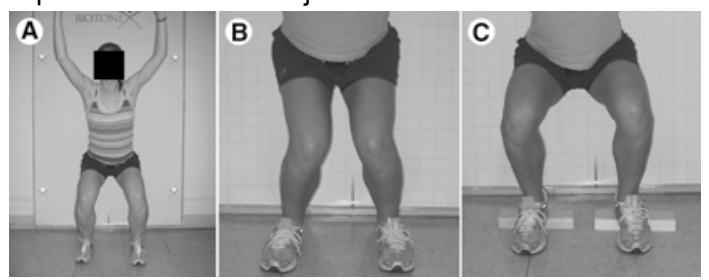

Figure 7. (a) Control subject during the overhead squat task. The mid-patella stays over the foot during the squat. (b) MKD during the overhead squat. The mid-patella is medial to great toe. (c) MKD subject performing the overhead squat on the heel-lift. MKD is corrected and mid-patella stays over the foot during the squat.
In a study by Bell et al, 2008, it was found that hip muscle weakness and tightness did not appear to be a factor contributing to excessive MKD. Findings suggested that weakness of the hip external rotators, gluteus maximus, and gluteus medius were not key factors contributing to excessive MKD.

It was posited that it was possible that participants with MKD may have had adequate hip strength, but not effective enough during the squat to prevent MKD. Likewise, 24 et al, when studying gluteal muscle activation in those with and without MKD during single leg squats, also came to the same conclusion. To support this, in a 2011 Bell study, in examining the strength ratios between the adductors and abductors, found that this co-activation ratio was not great enough to keep the knee out of valgus in the MKD group, and was approximately 1 . In contrast, the non-MKD group who maintained neutral alignment, had co-activation ratios of 3-4, indicating that the gluteal muscle activation was three to four times greater than that of the hip adductors. This indicates that gluteal activity can't be nearly equal with the hip adductor muscle activity. Similar findings were reported in research investigating a double-leg squat task(26), which found no significant differences in either gluteus maximus or gluteus medius muscle activation between those with and without visual presence of MKD.

However, there was significantly greater hip adductor muscle activation in the participants with MKD compared with those without the characteristic. It is worth noting that after ankle sprains, individuals will indeed display decreased gluteus maximus(5) or decreased gluteus medius muscle (6) activation.

Conversely, altered biomechanics due to a trauma to the foot-ankle complex will lead to decreased activation in the gluteals. These findings suggest the importance of restoring muscle balance between the hip abductors and adductors as part of knee injury prevention program. It may be necessary to address hip adductor over-activation by incorporating techniques that lead to inhibition and relaxation of the hip adductor muscles, as part of an integrated exercise strategy to restore muscle balance and minimize the hip adduction moment to produce increased MKD.

In conclusion, conditioning, rehabilitation, and injury prevention programs should focus on decreasing hip adductor activity, increasing hip abductor and external rotator activity, and increasing ankle dorsiflexion in striving to decrease the incidence of these injuries.

Common overuse ankle injuries to the recreational athlete due to overpronation

Overuse injuries refer to those sustained from repetitive action - as opposed to acute injuries, which occur in an instant (such as a sprained ankle).

Excessive and/or prolonged pronation of the foot puts it at increased risk of conditions that relate to biomechanical faults such as Achilles tendinitis, plantar fasciitis and shin splints.

Achilles tendonitis is a term that commonly refers to an inflammation of the Achilles tendon. It is an overuse injury that is common to joggers and jumpers. Most experts now use the term ,Achilles tendinopathy'. Most of these injuries occur during a sudden, abrupt lengthening (eccentric) phase of the calf muscles. In the background, there is a gradual degeneration and weakening of the tendon and its ability to lengthen further due to stiffness. An unusually strong contraction of the calf muscle can stretch the tendon to the point of tear or breaking. Stretching of the calf muscles is therefore very important before and after exercise along with eccentric strengthening of the tendon. 


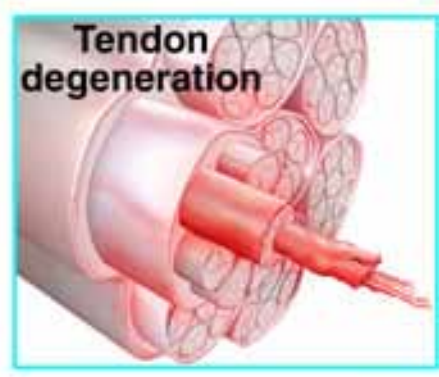

Achilles Tendonitis

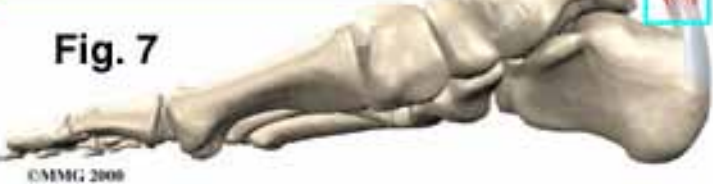

Figure 8: Achilles tendinopathy

The plantar fascia is a thick fibrous band of connective tissue originating along the bottom of the calcaneus (heel bone) and extends along the sole of the foot towards the toes. It acts as a passive limitation to the over-flattening of the arch. When the plantar fascia develops micro tears or becomes inflamed, it is known as plantar fasciitis. Flat feet or weak foot arch control muscles are two common causes of plantar fasciitis. To avoid such overuse injury, the arch muscles, which may be weak or have poor endurance, need to be exercised regularly. These foot muscles play a vital role as a stable base of your foot while also preventing excessive loading of your plantar fascia.

Shin splints are caused by overtraining of the muscles, which attach to the shin. It is associated with poor foot and leg biomechanics. Those with shin pain describe pain along the inside or front edges of the shin. The most common muscles that cause shin splints are anterior tibialis(anterior shin splints) and posterior tibialis (posterior shin splints) (Figure 9). As they are both responsible for inversion of the foot, after landing, excessive pronation makes their job very difficult, causing them to overstretch, overwork and inflame with time or with sudden increase of mileage or load. In addition, it is likely that the lateral calf muscles and the peroneals on the outside aspect of the ankle and lower leg become tight and overactive.

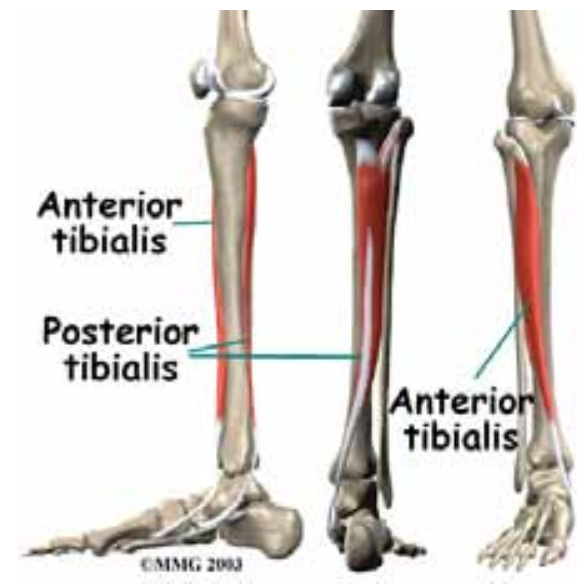

Figure 9. Anterior tibialis ad Posterior tibialis

\section{Static assessments of the foot and ankle}

When I was at university, one of the Gold Standard tests was the supine lying passive dorsiflexion ROM that was assessed under extended-knee and flexed-knee conditions. The degree of flexion was taken from 90 degrees flexed position of the foot with a goniometer (12). We were looking to achieve a minimum of 10 degrees in both feet, as we were taught that this was the range we all need to have to produce smooth, problem free walking.

It seems now that it is a more accurate and valid measurement when the subject is weight-bearing using forces equivalent to the body weight. However, to get a more valid measurement some using the weight bearing lunge test $(\mathrm{WBL})(2,10)$.

WBL test: Client stands with the right foot perpendicular to the wall, with the big toe $2.5 \mathrm{~cm}$ from the wall and knee in line with the second toe. With the right knee flexed, client attempts to touch the wall while keeping the entire foot flat on the ground paying specific attention to the heel. If successful, client moves $5 \mathrm{~cm}$ away from the wall and repeat the process until maximum distance is found. Goniometric measurement could be taken from the shin.

$\bullet<5 \mathrm{~cm}=$ failure of the test $\cdot 5-10 \mathrm{~cm}=$ acceptable range $\cdot 12.5+\mathrm{cm}$ inches $=$ optimal range

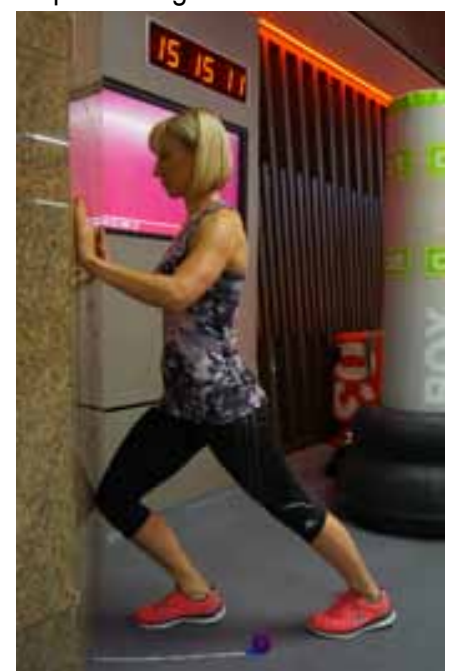

Figure 10. Weight bearing lunge test for measuring ankle dorsiflexion range

\section{Dynamic Assessments}

\section{Overhead Squat Test and Single Legged Squat Test (SLS)}

Restricted ankle range in the NASM overhead squat test and SLS test means that the person will show one or more of the following compensations: foot turns outward, flattening of the foot (pronation) or heel rising up and/or excessive forward lean from the hips. Having reduced ranges in DF means that functional activities such as squatting into a chair and running will require compensation from altered biomechanics. Squatting to 90 degrees or running requires approximately 20 degrees DF range, which I rarely seen in goniometric non-weight bearing tests with new clients. One of the most common things we see in people with a lack of dorsiflexion ROM is an "out-toeing," where the ankle opens up and allows the toes to move out.ln a more "uncompensated" scenario, an athlete with poor ankle mobility may push through the toe instead of the heel and the heel/s will come off the ground. Further up on the kinetic chain, poor ankle mobility causes the trunk to lean forward onto the thighs and there might be loss of the neutral spine present.

\section{TO BE CONTINUED..}




\section{References}

1. Azevedo L.B.,Lambert M.I., Vaughan C.L., O'Connor C.M., Schwellnus M.P. (2009): Biomechanical variables associated with Achilles tendinopathy in runners. Br J Sports Med. 43(4):288-92.

2. Backman, L.J., Danielson, P. (2011): Low range of ankle dorsiflexion predisposes for Patellar Tendinopathy in Junior Elite Basketball Players a 1-Year prospective study. Am J Sports Med. 39:2626-2633.

3. Bell D.R., Padua D.A., \& Clark M.A. (2008): Muscle strength and flexibility characteristics of people displaying excessive medial knee displacement. Arch Phys Med Rehabil 89(7):1323-1328.

4. Bell D.R., Vesci B.J., \&DiStefano L.J. (2011): Muscle activity and flexibility in individuals with medial knee displacement during the overhead squat. Athl Train Sports Health Care. 4(3):117-125. 20110817-03.

5. Bullock-Saxton J.E.,Janda V., \&Bullock M.I. (1994): The influence of ankle sprain injury on muscle activation during hip extension. Int J Sports Med. 15(6):330-4.

6. Claiborne T.L., Armstrong C.W., Gandhi V., Pincivero, D.M. (2006): Relationship between hip and knee strength and knee valgus during a single leg squat. J ApplBiomech. 22: 41-50

7. Clark M.A. \&Lucett S.C. (2011): NASM Essentials of Corrective Exercise Training. Lippincott Williams \& Wilkins, United States. pp252-265,280.

8. Davies, S.C., McBride, M., Keel, A., Hussey R. (2014): Musculoskeletal health: Public Health Approach. www. arthritisresearchuk.org/ /media/Files/.../public-health-guide.ashx 9. Devita P., \& Skelly W.A. (1992): Effect of landing stiffness on joint kinetics and energetics in the lower extremity. Med Sci Sports Exerc. 24(1):108-115.

10. Dill K.E.,Begalle R.L., Frank B.S., Zinder S.M., Padua D.A (2014): Altered knee and ankle kinematics during squatting in those with limited weight-bearing-lunge ankle-dorsiflexion range of motion. J Athl Train. 49(6):723-32.

11. Earl J.E. \&Hoch A.Z. (2011): A proximal strengthening program improves pain, function, and biomechanics in women with patellofemoral pain syndrome. Am J Sports Med. 39(1):154-63.

12. Fong C.M.,Blackburn J.T., Norcross M.F., McGrath M., Padua D.A. (2011): Ankle-dorsiflexion range of motion and landing biomechanics. J Athl Train. 46(1):5-10.

13. Graf A., Judge J.O. \&Ounpuu S. (2005): The effect of walking speed on lower-extremity joint powers among elderly adults who exhibit low physical performance. Arch Phys Med Rehabil. 86(11):2177-83

14. Hewett T.E. (2005): Anterior cruciate ligament injuries in female athletes: Part 2, a meta-analysis of neuromuscular interventions aimed at injury prevention. Am J Sports Med. 34(3):490-498.

15. Hirth C.J. \& Padua D.A. (2007): Clinical movement analysis to identify muscle imbalances and guide exercise. AthlTher Today. 12: $10-14$.

16. Holth H.S., Werpen H.K.B., Zwart J.A., Hagen K. (2008): Physical inactivity is associated with chronic musculoskeletal complaints 11 years later: Results from the Nord-Trondelag Health Study. BMC MusculoskelDisord. 9:159.

17. Huberti H.H. \&Hayes,W.C. (1984): Patellofemoral contact pressures(The influence of Q-angle and tendofemoral contact . J Bone Joint Surg Am. 66: 715-724.

18. Irving D.B.,Cook J.L, Menz H.B. (2006): Factors associated with chronic plantar heel pain: a systematic review. J Sci Med Sport. 9(1-2):11-22.
19. Jonkers I., Delp S., \& Patten C. (2009): Capacity to increase walking speed is limited by impaired hip and ankle power generation in lower functioning persons post-stroke. Gait Posture. 29(1):129-37.

20. Kendall F.P., McKCreary E.K. and Provance P.G. (2005): Muscle Testing and Function with Posture and Pain. 5th ed. Lippincott Williams and Wilkins, Baltimore, MD. pp19-22, 35, 5054, 410-411.

21. Lee T.Q., Yang B.Y., Sandusky M.D., McMahon P.J. (2001): The effects of tibial rotation on the patellofemoral joint: assessment of the changes in in situ strain in the peripatellar retinaculum and the patellofemoral contact pressures and areas. J Rehabil Res Dev. 38: 463-469.

22. Lubahn, A.J., Kernozek T. W., Tyson T.L.,Merkitch K.W. Reutemann P., Chestnut J.M. (2011): Hip muscle activation and knee frontal plane motion during weight bearing therapeutic exercises. Int J Sports PhysTher. 6(2): 92-103.

23. Macrum E.,Bell D.R., Boling M., Lewek M., Padua D. (2012): Effect of limiting ankle-dorsiflexion range of motion on lower extremity kinematics and muscle-activation patterns during a squat. J Sport Rehabil. 21(2):144-50.

24. Mauntel T.C.,Begalle R.L., Cram T.R., Frank B.S., Hirth C.J., Blackburn T., Padua D.A. (2013): The effects of lower extremity muscle activation and passive range of motion on single leg squat performance. J Strength Cond Res. 27(7):1813-23.

25. Mizuno Y., Kumagai M., Mattessich S.M. (2001): Q-angle influences tibiofemoral and patellofemoral kinematics. J Orthop Res. 19(5):834-840.

26. Padua D.A., Bell D.R., \& Clark M.A. (2012): Neuromuscular characteristics of individuals displaying excessive medial knee displacement. J Athl Train 47(5):525-536.

27. Page P., Clare C.F., and Ladner R. (2010): Assessment and Treatment of Muscle Imbalance. The Janda Approach. Human Kinetics, p89-90,98.

28. Paterno M.V., Schmitt L.C., Ford K.R. (2010): Biomechanical measures during landing and postural stability predict second anterior cruciate ligament injury after anterior cruciate ligament reconstruction and return to sport. Am J Sports Med. 38(10):19681978.

29. Powers C.M. (2003): The influence of altered lower-extremity kinematics on patellofemoral joint dysfunction: a theoretical perspective. J Orthop Sports PhysTher. 33(11):639-46.

30. Rao S., Riskowski J., Hannan M.T. (2012): Musculoskeletal Conditions of the Foot and Ankle: Assessments and Treatment Options. Best Pract Res ClinRheumatol. 26(3): 345-368.

31. Rees J.D.,Maffulli N., Cook J. (2009): Management of tendinopathy. Am J Sports Med. 37(9):1855-67.

32. Snyder K.R. Earl J.E.,O'Connor K.M., Ebersole K.T. (2008): Resistance training is accompanied by increases in hip strength and changes in lower extremity biomechanics during running. ClinBiomech. 24(1):26-34

33. Tweed J.L.,Campbell J.A., Avil S.J. (2008): Biomechanical risk factors in the development of medial tibial stress syndrome in distance runners. J Am Podiatr Med Assoc. 98(6):436-44. 34. Willson J.D., Ireland M.L., \& Davis, I. (2006): Core strength and lower extremity alignment during single leg squats. Med Sci Sports Exerc. 38: 945-952. 